\title{
Quantum Radiation Reaction and the Green's Function Decomposition
}

\author{
Atsushi Higuchi ${ }^{1}$ and Giles D. R. Martin ${ }^{2}$ \\ Department of Mathematics, University of York, Heslington, \\ York YO10 5DD, United Kingdom \\ email: ${ }^{1}$ ah28@york.ac.uk, ${ }^{2}$ gdrm100@york.ac.uk
}

(Dated: August 4, 2006)

\begin{abstract}
We analyze the change in position (the position shift) of the wave packet of a charged scalar particle due to radiation reaction in the $\hbar \rightarrow 0$ limit of quantum electrodynamics. In particular, we re-express the formula previously obtained for the position shift in terms of Green's functions for the electromagnetic field, thus clarifying the relation between the quantum and classical derivations of the radiation-reaction force.
\end{abstract}

PACS numbers: 03.65.-w,12.20.-m

\section{INTRODUCTION}

The interaction of a particle with its own field produces the phenomenon of radiation reaction. This phenomenon results in an extra force on the particle, a self-force, which changes the equations of motion. In classical electrodynamics in Minkowski spacetime this extra force is known as the Lorentz-Dirac force [1, 2, 3].

Since classical electrodynamics is the classical limit of quantum electrodynamics (QED), the Lorentz-Dirac force should be derived from QED in the $\hbar \rightarrow 0$ limit. This fact was demonstrated in Refs. [4, 5, 6] by showing that the change in position (the position shift) due to radiation reaction of a charged scalar particle in QED agrees with that in classical electrodynamics, with the Lorentz-Dirac force treated as a small perturbation, in the $\hbar \rightarrow 0$ limit. (See Refs. 7, 8, 9, 10, 11] for some other approaches to find the radiation-reaction force in QED.) In this Letter we analyze the expression for the quantum position shift obtained in Refs. 5, 6] to clarify the relation between the classical and quantum theories of radiation reaction. Our metric is $g_{\mu \nu}=\operatorname{diag}(+1,-1,-1,-1)$, and we use Greek letters for spacetime indices and Latin letters for space indices.

One of the methods for deriving the classical selfforce involves identification of the part of the self-field responsible for the force [3]. (This method has been extended to gravitational radiation [12].) For classical electrodynamics in Minkowski spacetime, the retarded Green's function $G_{-\mu \nu^{\prime}}\left(x-x^{\prime}\right)$ satisfies the equation $\partial_{\alpha} \partial^{\alpha} G_{-\mu \nu^{\prime}}\left(x-x^{\prime}\right)=\delta^{4}\left(x-x^{\prime}\right)$ and the boundary condition $G_{-\mu \nu^{\prime}}\left(x-x^{\prime}\right)=0$ for $x^{0}<x^{\prime 0}$, with primed indices corresponding to the primed spacetime coordinates. The $G_{-}$can be decomposed into two parts, the regular and singular Green's functions, $G_{\mathrm{R}}$ and $G_{\mathrm{S}}$ :

$$
G_{-\mu \nu^{\prime}}\left(x-x^{\prime}\right)=G_{R \mu \nu^{\prime}}\left(x-x^{\prime}\right)+G_{S \mu \nu^{\prime}}\left(x-x^{\prime}\right),
$$

with $G_{\mathrm{R}}=\frac{1}{2}\left(G_{-}-G_{+}\right)$and $G_{\mathrm{S}}=\frac{1}{2}\left(G_{-}+G_{+}\right)$, where $G_{+\mu \nu^{\prime}}\left(x-x^{\prime}\right)$ is the advanced Green's function. One shows that only the field generated by the regular Green's function $G_{R \mu \nu^{\prime}}\left(x-x^{\prime}\right)$ contributes to the radiation- reaction force, the field generated by the singular Green's function leading to an infinite correction to the inertial mass. Thus, the effective electromagnetic self-field contributing to the self-force is

$$
\begin{aligned}
A_{\mathrm{R} \mu}(x) & =\int d^{4} x^{\prime} G_{\mathrm{R} \mu}{ }^{\nu^{\prime}}\left(x-x^{\prime}\right) j_{\nu^{\prime}}\left(x^{\prime}\right), \\
j^{\nu}(x) & =e \frac{d x^{\nu}}{d t} \delta^{3}(\mathbf{x}-\mathbf{X}(t)),
\end{aligned}
$$

where $\mathbf{X}(t)$ is the position of the charged particle with charge $e$ at time $t$. [Here we are using the notation $x^{\mu}=(t, \mathbf{x})$.] Let $F_{\text {ext }}^{\mu \nu}$ be the external electromagnetic field that accelerates the charged particle with mass $m$. Then the equation of motion for this particle modified by the Lorentz-Dirac force is written as follows:

$$
\frac{d}{d t}\left(m \frac{d X^{\mu}}{d \tau}\right)=e F_{\mathrm{ext}}^{\mu \nu} \frac{d X^{\mu}}{d t}+f_{\mathrm{LD}}^{\mu},
$$

where

$$
f_{\mathrm{LD}}^{\mu}=e F_{\mathrm{R}}^{\mu \nu} \frac{d X_{\nu}}{d t},
$$

with $F_{\mathrm{R} \mu \nu} \equiv \partial_{\mu} A_{\mathrm{R} \nu}-\partial_{\nu} A_{\mathrm{R} \mu}$. Here $\tau$ denotes the propertime. Then it can be shown that

$$
f_{\mathrm{LD}}^{\mu}=\frac{e^{2}}{6 \pi}\left[\frac{d^{3} X^{\mu}}{d \tau^{3}}+\frac{d X^{\mu}}{d \tau}\left(\frac{d^{2} X^{\nu}}{d \tau^{2}} \frac{d^{2} X_{\nu}}{d \tau^{2}}\right)\right] \frac{d \tau}{d t} .
$$

(See, e.g. Refs. 13, 14, 15] for reviews.)

\section{CLASSICAL POSITION SHIFT}

(This and the next sections contain a summary of the relevant results obtained in Ref. [6].) In this Letter, the change in position of the charged particle due to radiation reaction, i.e. the position shift, plays the central role. We present here the position-shift formula in classical electrodynamics. Let the acceleration occur only for a finite time interval with $t<0$. The position shift, $\delta \mathbf{x}_{\mathrm{C}}$, that we consider is the position at $t=0$ of the particle satisfying 
Eq. (4) which would have passed through the origin at $t=0$ if $f_{\mathrm{LD}}^{\mu}$ were set to zero. Let us consider a family of trajectories $\mathbf{X}_{\mathbf{p}}(t)$ satisfying Eq. (4) with $f_{\mathrm{LD}}^{\mu}=0$ and the following conditions: (i) $\mathbf{X}_{\mathbf{p}}(0)=0$, (ii) the momentum of the particle at $t=0$ is $\mathbf{p}$. Then, the position shift can readily be found with $f_{\mathrm{LD}}^{\mu}$ treated as a small perturbation using the idea of "reduction of order" [16, 17] to first order in $f_{\mathrm{LD}}^{\mu}$ :

$$
\delta x_{\mathrm{C}}^{i}=-\int_{-\infty}^{0} d t f_{\mathrm{LD}}^{j}(t) \frac{\partial X_{\mathbf{p}}^{j}(t)}{\partial p^{i}},
$$

where $f_{\mathrm{LD}}^{\mu}$ is evaluated on the unperturbed trajectory and where $t$ is held fixed in the partial derivative with respect to $p^{i}$. Substituting Eq. (5) we have (with $X_{\mathbf{p}}^{0}(t) \equiv$ t)

$$
\delta x_{\mathrm{C}}^{i}=e \int_{-\infty}^{0} d t F_{\mathrm{R} \mu \nu} \frac{d X_{\mathbf{p}}^{\nu}}{d t} \frac{\partial X_{\mathbf{p}}^{\mu}}{\partial p^{i}}
$$

\section{QUANTUM POSITION SHIFT}

In this section we present the formula for the quantum position shift. The quantum-field-theory model corresponding to the classical model in the previous section is the wave packet of a charged scalar particle accelerated by an external electromagnetic field, which is assumed to be nonzero only for a finite time in the past of the $t=0$ hypersurface. We assume that the external potential $A_{\text {ext }}^{\mu}$ (with $A_{\text {ext }}^{0}=0$ ) depends only on time $t$, and that $A_{\text {ext }}^{i}=0$ for $t>0$. Then the momentum of the scalar particle is conserved (in the absence of radiation reaction). For this reason we label the mode functions of the quantum scalar field, chosen to have the form $e^{-i k \cdot x}$ for $t>0$, by the momentum p. [Note, however, that the proper-velocity $d x^{i} / d \tau$ of the particle is $\left(p^{i}-A_{\text {ext }}^{i}\right) / m$ rather than $p^{i} / m$.] Let $|\mathbf{p}\rangle$ be the scalar one-particle state with momentum p. When the electromagnetic interaction is turned on, to order $e^{2}$ this state evolves in general as

$$
\begin{aligned}
|\mathbf{p}\rangle \rightarrow & {[1+i \mathcal{F}(\mathbf{p})]|\mathbf{p}\rangle } \\
+ & \frac{i}{\hbar} \int \frac{d^{3} \mathbf{k}}{(2 \pi)^{3} 2 k} \mathcal{A}^{\mu}(\mathbf{p}, \mathbf{k}) \hat{a}_{\mu}^{\dagger}(\mathbf{k})|\mathbf{P}\rangle,
\end{aligned}
$$

with $k \equiv\|\mathbf{k}\|$ and $\mathbf{P}=\mathbf{p}-\hbar \mathbf{k}$, where the annihilation and creation operators for the photons, $\hat{a}_{\mu}(\mathbf{k})$ and $\hat{a}_{\mu}^{\dagger}(\mathbf{k})$, satisfy in the Feynman gauge

$$
\left[\hat{a}_{\mu}(\mathbf{k}), \hat{a}_{\nu}^{\dagger}\left(\mathbf{k}^{\prime}\right)\right]=-g_{\mu \nu}(2 \pi)^{3} 2 \hbar k \delta^{3}\left(\mathbf{k}-\mathbf{k}^{\prime}\right) .
$$

The amplitudes $\mathcal{F}(\mathbf{p})$ and $\mathcal{A}^{\mu}(\mathbf{p}, \mathbf{k})$ are the forwardscattering and (one-photon-)emission amplitudes, respectively.

The position expectation value of a wave-packet state $|w\rangle$ of the scalar particle is defined by

$$
\left\langle x^{i}\right\rangle \equiv \int d^{3} \mathbf{x} x^{i}\left\langle w\left|\hat{J}^{0}\right| w\right\rangle,
$$

where $\hat{J}_{\mu}=(i / \hbar): \hat{\varphi}^{\dagger} \partial_{\mu} \hat{\varphi}-\partial_{\mu} \hat{\varphi}^{\dagger} \cdot \hat{\varphi}:$ is the normalordered current density operator for the charged scalar field $\hat{\varphi}$. The $e^{2}$ contribution to this quantity is the quantum position shift $\delta \mathbf{x}_{\mathrm{Q}}$. This quantity is found in the limit $\hbar \rightarrow 0$ as

$$
\begin{aligned}
\delta x_{\mathrm{Q}}^{i}= & -\frac{i}{2} \int \frac{d^{3} \mathbf{k}}{2 k(2 \pi)^{3}} \mathcal{A}^{\mu *}(\mathbf{p}, \mathbf{k}) \stackrel{\leftrightarrow}{\partial}_{p^{i}} \mathcal{A}_{\mu}(\mathbf{p}, \mathbf{k}) \\
& -\hbar \partial_{p^{i}} \operatorname{Re} \mathcal{F}(\mathbf{p}) .
\end{aligned}
$$

In Ref. [6] the right-hand side was shown to be equal to the classical position shift, $\delta x_{\mathrm{C}}^{i}$, given by Eq. (7) with $f_{\mathrm{LD}}^{\mu}$ found in Eq. (6). This calculation did not give much mathematical insight into why the quantum position shift in the $\hbar \rightarrow 0$ limit is identical to the classical one. In this Letter we express the quantum position shift given by Eq. (12) in terms of Green's functions and clarify the reason why it agrees with the classical expression. In the next two sections we shall analyze the contribution from the emission amplitude and that from the forward scattering amplitude in Eq. (12) in turn.

\section{CONTRIBUTION FROM THE EMISSION AMPLITUDE}

The emission contribution to the quantum position shift (12) is

$$
\delta x_{\mathrm{em}}^{i}=-\frac{i}{2} \int \frac{d^{3} k}{2 k(2 \pi)^{3}} \mathcal{A}^{\mu *}(\mathbf{p}, \mathbf{k}) \stackrel{\leftrightarrow}{\partial}_{p^{i}} \mathcal{A}_{\mu}(\mathbf{p}, \mathbf{k}) .
$$

Using the WKB scalar mode functions in the presence of external field, one finds that the emission amplitude coincides with that from a classical point charge to order $\hbar^{0}$ [22]. That is [6],

$$
\mathcal{A}^{\mu}(\mathbf{p}, \mathbf{k})=-\int_{-\infty}^{+\infty} e^{i k \cdot x} j^{\mu}(x),
$$

where the current $j^{\mu}(x)$ is given by

$$
j^{\mu}(x)=\frac{d x^{\mu}}{d t} \delta^{3}\left(\mathbf{x}-\mathbf{X}_{\mathbf{p}}(t)\right) \chi(t) .
$$

Here the function $\chi(t)$ is a cut-off factor that takes the value 1 when the external force is nonzero and smoothly becomes zero for large $|t|$. The classical field emitted from the current (15) is

$$
A_{-}^{\mu}(x)=\int d^{4} x^{\prime} G_{-}^{\mu}{ }_{\nu^{\prime}}\left(x-x^{\prime}\right) j^{\nu^{\prime}}\left(x^{\prime}\right) .
$$

Now, the retarded Green's function can be written as 18]

$$
\begin{aligned}
G_{-\mu \nu^{\prime}}\left(x-x^{\prime}\right)=i g_{\mu \nu^{\prime}} \theta\left(t-t^{\prime}\right) \int \frac{d^{3} \mathbf{k}}{2 k(2 \pi)^{3}} \\
\times\left[e^{-i k \cdot\left(x-x^{\prime}\right)}-e^{i k \cdot\left(x-x^{\prime}\right)}\right] .
\end{aligned}
$$


These two equations and Eq. (15) imply that

$A_{-}^{\mu}(x)=-i \int \frac{d^{3} k}{2 k(2 \pi)^{3}}\left[\mathcal{A}^{\mu}(\mathbf{p}, \mathbf{k}) e^{-i k \cdot x}-\mathcal{A}^{\mu *}(\mathbf{p}, \mathbf{k}) e^{i k \cdot x}\right]$,

for large enough $t$ such that $\chi(t)=0$. Then, by a straightforward calculation one finds that the emission contribution to the position shift given by Eq. (13) can be expressed as follows:

$$
\delta x_{\mathrm{em}}^{i}=-\frac{1}{2} \int_{t=T} d^{3} \mathbf{x}\left(\partial_{p^{i}} A_{-}^{\mu}\right) \stackrel{\leftrightarrow}{\partial}_{t} A_{-\mu},
$$

where $t=T$ is far into the future so that $\chi(T)=0$. By substituting Eq. (16) and using

$$
\begin{aligned}
\int_{t=T} d^{3} \mathbf{x} G_{-}{ }^{\mu}{ }_{\alpha^{\prime \prime}}\left(x-x^{\prime \prime}\right) & \stackrel{\leftrightarrow}{\partial}_{t} G_{-\mu \beta^{\prime}}\left(x-x^{\prime}\right) \\
& =-2 G_{\mathrm{R} \alpha^{\prime \prime} \beta^{\prime}}\left(x^{\prime \prime}-x^{\prime}\right),
\end{aligned}
$$

for $x_{0}>\max \left(x_{0}^{\prime}, x_{0}^{\prime \prime}\right)$, we obtain

$$
\begin{aligned}
\delta x_{\mathrm{em}} & =\int d^{4} x d^{4} x^{\prime} \partial_{p^{i}} j_{\mu}(x) G_{R}^{\mu \nu^{\prime}}\left(x-x^{\prime}\right) j_{\nu^{\prime}}\left(x^{\prime}\right) \\
& =\int d^{4} x \partial_{p^{i}} j^{\mu}(x) A_{\mathrm{R} \mu}(x),
\end{aligned}
$$

where the regular field $A_{\mathrm{R}}^{\mu}(x)$ is defined by Eq. (2). By a calculation analogous to the derivation of the Lorentz force from the standard Lagrangian for a point charge in an external electromagnetic field (see, e.g. Ref. [15]), we find from Eq. (21)

$$
\begin{aligned}
\delta x_{\mathrm{em}}^{i} & =e \int d t F_{\mathrm{R} \mu \nu} \frac{d X_{\mathrm{p}}^{\nu}}{d t}\left(\frac{\partial X_{\mathbf{p}}^{\mu}}{\partial p^{i}}\right)_{t} \\
& =-e \int_{-\infty}^{0} d t f_{\mathrm{LD}}^{j}\left(\frac{\partial X_{\mathbf{p}}^{j}}{\partial p^{i}}\right)_{t} .
\end{aligned}
$$

Surface terms can be dropped thanks to the cut-off function $\chi(t)$. The final result is independent of the choice of $\chi(t)$. We have made the upper bound of the $t$-integration to $t=0$ in the last line because $f_{\mathrm{LD}}^{j}=0$ for $t>0$. Thus, we have shown that the contribution from the emission of a photon to the position shift agrees with the classical counterpart using the Green's function method.

\section{CONTRIBUTION FROM THE FORWARD-SCATTERING AMPLITUDE}

We now turn to the forward-scattering contribution to the position shift given by

$$
\delta x_{\text {for }}^{i}=-\hbar \partial_{p^{i}} \operatorname{Re} \mathcal{F}(\mathbf{p}) .
$$

This contribution vanishes in the end [6]. More precisely, the leading order terms of the real part of the forwardscattering amplitude are exactly cancelled by the contribution from the mass counter-term, i.e. it is eliminated to

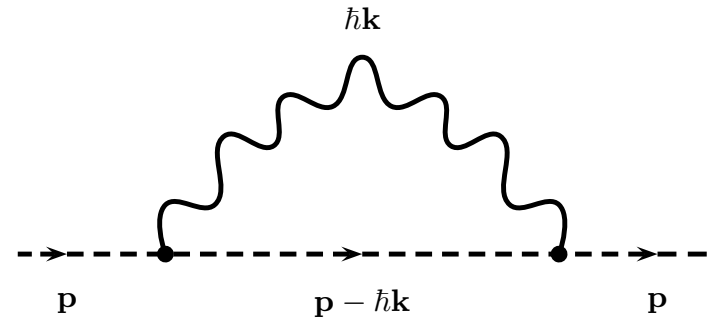

FIG. 1: The one-loop diagram contributing to the forwardscattering amplitude: the dashed and wavy lines represent the scalar and photon propagators, respectively.

order $\hbar^{0}$ by the mass renormalization. Here we shall see that the field generated by the singular Green's function appears in the calculation of $\delta x_{\text {for }}^{i}$.

The forward-scattering amplitude comes from the oneloop diagram shown in Fig. 1 (and the tadpole diagram, which can be ignored in the end). For the contribution from the intermediate particle state (as opposed to antiparticle state), we divide the momentum integral for the virtual photon in this loop diagram into two parts; one with momentum $\hbar\|\mathbf{k}\|$ less than $\hbar^{\alpha} \lambda$ and the other with momentum larger than $\hbar^{\alpha} \lambda$, where $\alpha$ and $\lambda$ are constants. We choose $\alpha$ to satisfy $\frac{3}{4}<\alpha<1$ as in Ref. [6], although the condition $\alpha<1$ will suffice for the purpose of this Letter. Denoting the first part with the virtual-photon momentum below the cut-off by $\mathcal{F}^{<}(\mathbf{p})$, we find to lowest order in $\hbar[\underline{6}]$

$$
\begin{aligned}
\hbar \mathcal{F}^{<}(\mathbf{p})= & -i e^{2} \int_{k \leq \hbar^{\alpha-1} \lambda} \frac{d^{3} \mathbf{k}}{(2 \pi)^{3} 2 k} \int_{-\infty}^{+\infty} d t \int_{-\infty}^{+\infty} d t^{\prime} \\
& \times \theta\left(t-t^{\prime}\right) \frac{d X_{\mathbf{p}}^{\mu}}{d t} \frac{d X_{\mathbf{p} \mu}}{d t^{\prime}} e^{i k\left(t^{\prime}-t\right)-i \mathbf{k} \cdot\left(\mathbf{X}_{\mathbf{p}}\left(t^{\prime}\right)-\mathbf{X}_{\mathbf{p}}(t)\right)} .
\end{aligned}
$$

Here we have corrected some misprints in the corresponding equation, Eq. (A37), in Ref. [6]. In the classical limit $\hbar \rightarrow 0$, the $\mathbf{k}$-integration will have no restriction because $\hbar^{\alpha-1} \lambda \rightarrow \infty$. Recalling that the Feynman propagator is given by

$$
\begin{aligned}
G_{F}^{\mu \nu^{\prime}}\left(x-x^{\prime}\right)= & -i \hbar g^{\mu \nu^{\prime}} \int \frac{d^{3} k}{(2 \pi)^{3} 2 k} \\
& \times\left[\theta\left(x_{0}-x_{0}^{\prime}\right) e^{-i k \cdot\left(x-x^{\prime}\right)}+\left(x \leftrightarrow x^{\prime}\right)\right],
\end{aligned}
$$

we find in this limit

$$
\hbar \mathcal{F}^{<}(\mathbf{p})=\frac{1}{2 \hbar} \int d^{4} x d^{4} x^{\prime} j_{\mu}(x) j_{\nu^{\prime}}\left(x^{\prime}\right) G_{F}^{\mu \nu^{\prime}}\left(x-x^{\prime}\right) .
$$

Let us write Feynman propagator as the sum of the real and imaginary parts:

$$
G_{F}^{\mu \nu^{\prime}}\left(x-x^{\prime}\right)=-\hbar G_{S}^{\mu \nu^{\prime}}\left(x-x^{\prime}\right)-i \hbar G^{(1) \mu \nu^{\prime}}\left(x-x^{\prime}\right),
$$


where

$$
\hbar G^{(1) \mu \nu^{\prime}}\left(x-x^{\prime}\right)=\left\langle 0\left|\left\{\hat{A}^{\mu}(x), \hat{A}^{\nu^{\prime}}\left(x^{\prime}\right)\right\}\right| 0\right\rangle,
$$

with $\hat{A}^{\mu}(x)$ being the quantum electromagnetic potential. The imaginary part $\operatorname{Im} \mathcal{F}^{<}(\mathbf{p})$ equals half the emission probability as required by unitarity:

$$
\operatorname{Im} \mathcal{F}^{<}(\mathbf{p})=-\frac{1}{2 \hbar} \int \frac{d^{3} \mathbf{k}}{2 k(2 \pi)^{3}} \mathcal{A}_{\mu}^{*}(\mathbf{p}, \mathbf{k}) \mathcal{A}^{\mu}(\mathbf{p}, \mathbf{k}) .
$$

(It turns out that the contribution to the forwardscattering amplitude above the cut-off has no imaginary part in the $\hbar \rightarrow 0$ limit [6] ). Using the symmetry of $G_{S}^{\mu \nu^{\prime}}\left(x-x^{\prime}\right)$, we obtain

$$
-\hbar \partial_{p^{i}} \operatorname{Re} \mathcal{F}^{<}(\mathbf{p})=\int d^{4} x \partial_{p^{i}} j^{\mu}(x) A_{S \mu}(x),
$$

where $A_{S}^{\mu}(x)$ is the singular part of the self-field given by

$$
A_{S}^{\mu}(x)=\int d^{4} x^{\prime} G_{S}^{\mu \nu^{\prime}}\left(x-x^{\prime}\right) j_{\nu^{\prime}}\left(x^{\prime}\right) .
$$

If we add Eq. (30) to the emission contribution, i.e. Eq. (21), then

$$
\delta x_{\mathrm{em}}-\hbar \partial_{p^{i}} \operatorname{Re} \mathcal{F}^{<}(\mathbf{p})=\int d^{4} x \partial_{p^{i}} j_{\mu}(x) A_{-}^{\mu}(x),
$$

where the self-field, $A_{-}^{\mu}(x)$, is given by Eq. (16). Thus, the one-photon emission process and the low-energy part of the forward-scattering process are incorporated in the classical self-field $A_{-}^{\mu}(x)$ if one sees this field from the viewpoint of quantum derivation of the self-force. The regular part, $A_{R}^{\mu}(x)$, of the self-field in classical electrodynamics corresponds to the emission process in QED and the singular part, $A_{S}^{\mu}(x)$, to the low-energy forwardscattering process. The remaining high-energy and intermediate anti-particle state contributions to the forwardscattering amplitude in QED have no classical counterpart. The forward-scattering contribution as a whole vanishes if one includes the quantum mass counter-term, as was shown in Ref. [6].

\section{SUMMARY}

In this Letter we re-expressed the $\hbar \rightarrow 0$ limit of the position shift due to radiation reaction in QED, Eq. (12), in terms of the Green's functions for the electromagnetic field, thus making the relation between the classical and quantum derivations of the radiation-reaction force more transparent. We believe that the insight gained in this Letter will be useful in finding the electomagnetic and gravitational radiation-reaction forces in quantum field theory in curved spacetime, which are to be compared with results in classical field theory in the literature [19, 20, 21].

[1] M. Abraham and R. Becker, Theorie der Elektrizität, Vol. II, (Springer, Leipzig, 1933).

[2] H. A. Lorentz, Theory of electrons, (Dover, New York, 1952).

[3] P. A. M. Dirac, Proc. Roy. Soc. London A167, 148 (1938).

[4] A. Higuchi, arXiv: quant-ph/9812036 Phys. Rev. D 66, 105004 (2002); Erratum ibid. 69, 129903 (2004), arXiv: quant-ph/0208017

[5] A. Higuchi and G. D. R. Martin, Phys. Rev. D, 70, 081701(R) (2004), arXiv: quant-ph/0407162 Found. Phys. 35, 1572 (2005), arXiv: quant-ph/0501026

[6] A. Higuchi and G. D. R. Martin, Phys. Rev. D, 73, 025019 (2006), arXiv: quant-ph/0510043

[7] E. J. Moniz and D. H. Sharp, Phys. Rev. D 10, 1133 (1974); ibid. 15, 2850 (1977).

[8] P. R. Johnson and B. L. Hu, Phys. Rev. D 65, 065015 (2002), arXiv: quant-ph/0101001

[9] V. S. Krivitskiî and V. N. Tsytovich, Sov. Phys. Usp. 34, 250 (1991).

[10] G. W. Ford and R. F. O'Connell, Phys. Lett. A157, 217 (1991).

[11] R. F. O'Connell, Phys. Lett. A313, 491 (2003).

[12] S. Detweiler and B. F. Whiting, Phys. Rev. D 67, 024025 (2003), ArXiv: gr-qc/0202086

[13] E. Poisson, An introduction to the Lorentz-Dirac equation, arXiv:gr-qc/9912045 Class. Quantum Grav. 21, R153 (2004)

[14] F. T. Rohrlich, Classical charged particles, (AddisonWesley, Reading, Mass., 1965).

[15] J. D. Jackson, Classical electrodynamics, (Wiley, New York, 1975).

[16] L. D. Landau and E. M. Lifshitz, The classical theory of fields, (Pergamon, Oxford, 1962).

[17] É. É. Flanagan and R. M. Wald, Phys. Rev. D 54, 6233 (1996), arXiv: gr-qc/9602052

[18] C. Itzykson and J.-B. Zuber, Quantum field theory, (McGraw-Hill, New York, 1980).

[19] Y. Mino, M. Sasaki and T. Tanaka, Phys. Rev. D 55, 3457 (1997), arXiv: gr-qc/9606018

[20] T. C. Quinn and R. M. Wald, Phys. Rev. D 56, 3381 (1997), arXiv: gr-qc/9610053

[21] T. C. Quinn, Phys. Rev. D 62, 064029 (2000), arXiv: gr-qc/0005030

[22] This is the case if the external electromagnetic potential depends only on one time or space coordinate [6]. 